\title{
Safety and Efficacy of Epithelium-On Corneal Collagen Cross-Linking Using a Multifactorial Approach to Achieve Proper Stromal Riboflavin Saturation
}

\author{
Aleksandar Stojanovic, ${ }^{1,2}$ Xiangjun Chen, ${ }^{2}$ Nan Jin, ${ }^{3}$ Ting Zhang, ${ }^{3}$ \\ Filip Stojanovic, ${ }^{4}$ Sten Raeder, ${ }^{5,6}$ and Tor Paaske Utheim ${ }^{2,7}$ \\ ${ }^{1}$ Department of Ophthalmology, University Hospital of North Norway, Fløyvn. 32, Tromsdalen, 9020 Tromsø, Norway \\ ${ }^{2}$ SynsLaser Kirurgi, 9008 Tromsø and 0159 Oslo, Norway \\ ${ }^{3}$ School of Ophthalmology and Optometry and Eye Hospital, Wenzhou Medical College, Zhejiang, Wenzhou 325003, China \\ ${ }^{4}$ University of Tromsø, 9037 Tromsø, Norway \\ ${ }^{5}$ Department of Ophthalmology, Stavanger University Hospital, 4011 Stavanger, Norway \\ ${ }^{6}$ Department of Clinical Medicine, University of Bergen, 5020 Bergen, Norway \\ ${ }^{7}$ Department of Medical Biochemistry, Oslo University Hospital, 0424 Oslo, Norway
}

Correspondence should be addressed to Aleksandar Stojanovic, aleks@online.no

Received 3 February 2012; Accepted 10 June 2012

Academic Editor: Andrew G. Lee

Copyright ( $) 2012$ Aleksandar Stojanovic et al. This is an open access article distributed under the Creative Commons Attribution License, which permits unrestricted use, distribution, and reproduction in any medium, provided the original work is properly cited.

Purpose. To evaluate the efficacy and safety of epithelium-on corneal collagen cross-linking (CXL) using a multifactorial approach to achieve proper stromal riboflavin saturation. Methods. This non-randomized retrospective study comprised 61 eyes with progressive keratoconus treated with epithelium-on CXL. Chemical epithelial penetration enhancement (benzalkonium chloride-containing local medication and hypotonic riboflavin solution), mechanical disruption of the superficial epithelium, and prolongation of the riboflavin-induction time until verification of stromal saturation were used before the UVA irradiation. Uncorrected and corrected distance visual acuity (UDVA, CDVA), refraction, corneal topography, and aberrometry were evaluated at baseline and at 1,3,6, and 12 months postoperative. Results. At 12-month, UDVA and CDVA improved significantly. None of the eyes lost lines of CDVA, while $27.4 \%$ of the eyes gained 2 or more lines. Mean spherical equivalent decreased by $0.74 \mathrm{D}$, and mean cylindrical reduction was $1.15 \mathrm{D}$. Irregularity index and asymmetry from Scheimpflug-based topography and Max- $K$ at the location of cone from Placido-based topography showed a significant decrease. Higher-order-aberration data demonstrated a slight reduction in odd-order aberrations $S 3,5,7(P=0.04)$. Postoperative pain without other complications was recorded. Conclusion. Epithelium-on CXL with our novel protocol appeared to be safe and effective in the treatment of progressive keratoconus.

\section{Introduction}

Corneal collagen cross-linking (CXL) is a low-invasive treatment aimed to improve biomechanical stability in eyes with keratectasia [1-3]. A photodynamic reaction induced by photosensitizing riboflavin and ultraviolet A (UVA) light causes an increase of the number of intra- and interfibrillar covalent bonds and the corneal collagen resistance against enzymatic degradation [4-6]. Increased stromal biomechanical strength and lamellar compaction lead to stabilized corneal shape and better corneal symmetry, potentially causing an improvement in visual function $[2,7-9]$.

In CXL, riboflavin has a dual function acting both as a photosensitizer inducing the physical collagen cross-linking and as an absorber of the UVA irradiation, preventing damage to deeper ocular structures $[10,11]$. Proper corneal stromal saturation with riboflavin is therefore essential in CXL, and without its presence the UVA radiation may cause the collagen fibers to degrade rather than to facilitate crosslinking [12]. 
The "standard CXL protocol" described by Wollensak and colleagues includes removal of the corneal epithelium in a diameter of $9 \mathrm{~mm}$, followed by saturation of the corneal stroma using $0.1 \%$ isotonic riboflavin solution in $20 \%$ dextran [13]. This procedure is proved to be effective in increasing corneal stiffness [13], stabilization of keratoconus, and in some cases in improving the refractive and topographic features $[14,15]$. Even so, the epithelial removal may lead to serious complications that include infection [16, 17], stromal haze [18], and corneal melting [19] in addition to severe pain and decrease in vision occurring during the first days after the treatment. To avoid such complications, Boxer Wachler et al. suggested a modification of the technique by keeping the epithelium intact (epithelium-on or transepithelial CXL) [20]. However, finding appropriate means of increasing corneal epithelial permeability prior to riboflavin application was warranted as riboflavin has a molecular weight of $338 \mathrm{Da}$, whereas the corneal epithelium is impermeable to compounds with a molecular weight greater than $100 \mathrm{Da}$ [21]. Accordingly, various approaches have been tried clinically and in the laboratory to enhance the epithelial permeability before the riboflavin application. Chemical enhancers such as benzalkonium chloride (BAC), ethylenediaminetetraacetic acid (EDTA), gentamycin, tetracaine, and 20\% ethanol [2225] were used, as well as partial grid-like pattern deepithelialization [22], excimer laser superficial epithelial removal [26], and the replacement of the isotonic by hypotonic riboflavin solution $[24,27]$. The results varied between the studies, but the majority of the aforementioned methods lead to increased epithelial permeability for riboflavin.

In the current study, a multifactorial approach was utilized to enhance the riboflavin penetration by employing: (1) BAC-containing local medication; (2) hypotonic riboflavin solution without dextran; (3) increased riboflavin solution concentration; (4) mechanical disruption of the superficial epithelium (microabrasions); (5) prolongation of the riboflavin-induction time until objective verification of the stromal saturation is confirmed. By such an approach, this nonrandomized retrospective study aimed to evaluate the efficacy and safety of the epithelium-on CXL in treatment of progressive keratoconus.

\section{Patients and Methods}

In this retrospective, interventional case series, we reviewed the medical records of all patients with advanced progressive keratoconus who had 12-month observation time after the epithelium-on CXL treatment using our multifactorial approach. The treatment was performed at The Eye Department of the University Hospital North Norway, Tromsø, Norway, between September 15, 2009 and September 15, 2010. This study was approved by the regional ethics committee and adhered to the official ethical regulations for clinical research and the Tenets of the Declaration of Helsinki. Inclusion criteria included (1) documented progression of keratoconus during the last 12 months before treatment (increase of astigmatism or myopia by $1.00 \mathrm{D}$ or increase in average SimK by $1.50 \mathrm{D}$ ); (2) minimum corneal thickness of no less than $400 \mu \mathrm{m}$ at the thinnest point measured by ultrasound pachymetry; (3) age ranging from 18 to 45 years; (4) Amsler-Krumeich keratoconus classification stage II to III. Exclusion criteria were: (1) history of herpes virus keratitis; (2) severe dry eye; (3) concurrent corneal infections; (4) previous ocular surgery; (5) hard contact lens wear $\leq 4$ weeks before the baseline examination.

Pre- and postoperative assessments consisted of slit lamp biomicroscopy, Scheimpflug-based corneal topo/tomography (Precisio, iVIS Technology, Taranto, Italy), Placido disk-based topography and wavefront aberrometry (OPD-Scan II, Nidek. Co., Ltd. Aichi, Japan), uncorrected (UDVA) and corrected (CDVA) distance visual acuities (Nidek RT 2100 system, Nidek Co. Ltd., Aichi, Japan), ultrasound pachymetry (Cornea-Gage Plus, Sonogage Inc., Cleveland, Ohio), tonometry (Icare tonometer, Revenio Group Corporation, Helsinki, Finland), and patients' subjective evaluation of postoperative pain. The patients were examined at 1,3,6, and 12 months postoperative.

2.1. Surgical Technique. To reduce the risk for UV exposure of retroiridal eye structures, miosis was induced by applying two drops of pilocarpine 2\% (Pilokarpin, Ophtha AS, Norway). It was followed by the application of two drops of local anesthetic proparacaine $0.5 \%$ (Alcaine, Alcon Norway AS), two drops of local antibiotic gentamycin 0.3\% (Garamycin, Schering-Plough AS, Norway) followed by proparacaine again, one drop every minute for five minutes. All the drops were preserved by BAC $(0.001 \%$ for Pilokarpin, $0.005 \%$ for Garamycin and $0.01 \%$ for Alcaine), aiming to increase the epithelial permeability by chemically disrupting the tight junction proteins. A round Merocel sponge (Medtronic, Inc., Minneapolis, MN) of $5 \mathrm{~mm}$ in diameter was inserted into the conjunctival sac to provide a depot of riboflavin, and to produce microabrasions of the superficial epithelial layers caused by friction upon patient's blinking. Thereafter, two drops of proparacaine and two drops of $0.5 \%$ aqueous riboflavin solution without dextran (Vitamin B2; Streuli, Uznach, Switzerland) were applied alternating every 30 seconds, until the riboflavin saturation was verified by the slit-lamp inspection of the cornea and by the determination of the presence of riboflavin flare in the anterior chamber (Figure 1). Under the same examination the staining of the epithelial microabrasions was verified. The initial slitlamp saturation evaluation was performed 25 minutes after the first application of riboflavin and repeated every five minutes until the saturation was confirmed. During the premedication and riboflavin induction time the patient was instructed to blink normally between eye drop instillation and to remain in a comfortable sitting position. The Merocel sponge was then removed and corneal thickness measured with ultrasound pachymetry, at which point the patient was placed in supine position. Irrigation with isotonic balanced salt solution (BSS) was performed before the UVA irradiation in order to avoid the shielding effect of riboflavin covering the epithelium. An eyelid speculum was then inserted, and a ring-shaped Merocel shield k20-5021 (Katena Products, Inc. 


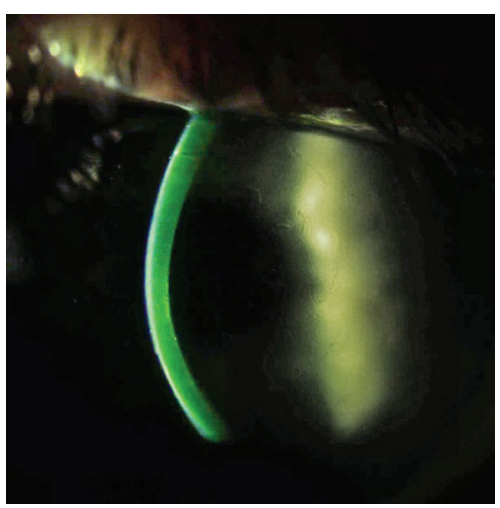

(a)

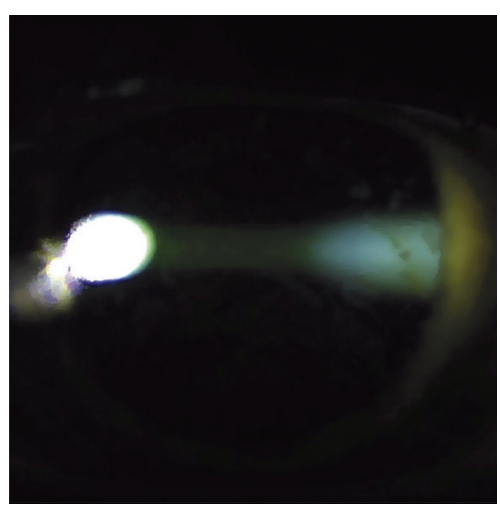

(b)

FIGURE 1: . Slit lamp verification of the stromal riboflavin saturation before the UVA irradiation.

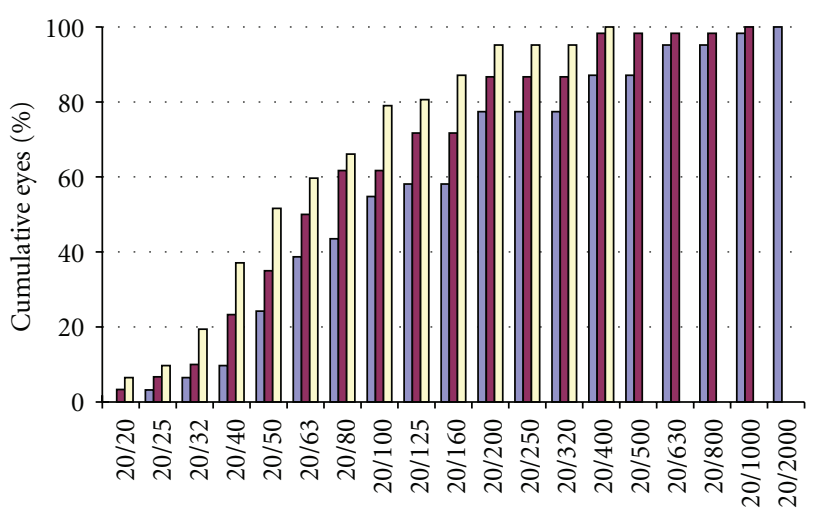

Cumulative Snellen visual acuity $(20 / X)$

a $\operatorname{Preop}(n=61)$

- $1 \mathrm{~m}$ postop $(n=59)$

ㅁ $12 \mathrm{~m}$ postop $(n=61)$

FIGURE 2: UDVA 1 months and 12-months after the epithelium-on CXL.

Denville, NJ) was applied to protect the limbal region and its stem cells from UVA radiation.

The cornea was subjected to UVA radiation for 30 minutes with a wavelength of $365 \mathrm{~nm}$ at a working distance of $5 \mathrm{~cm}$. The UV-X lamp (IROC AG, Zürich, Switzerland) provided an irradiance of $3 \mathrm{~mW} / \mathrm{cm}^{2}$ within a circular diameter of $9 \mathrm{~mm}$. During the irradiation, BSS was applied every three minutes, and proparacaine drops were added as needed.

After the UVA irradiation, two drops of atropine 1\% (Atropin minims, Chauvin, England) and 2 drops of gentamycin were applied. The cornea was protected with a soft bandage contact lens for 12-18 hours. Instructions were given to apply a mixture of $0.1 \%$ dexamethasone and $0.5 \%$ chloromycetin (Spersadex med Kloramfenikol, Novartis, Norway) eye drops four times daily for seven days, as well as to use artificial tears as needed.

2.2. Statistical Analysis. All visual acuity values were recorded as Snellen values, converted to LogMAR for statistical analyses and then changed back to Snellen values for

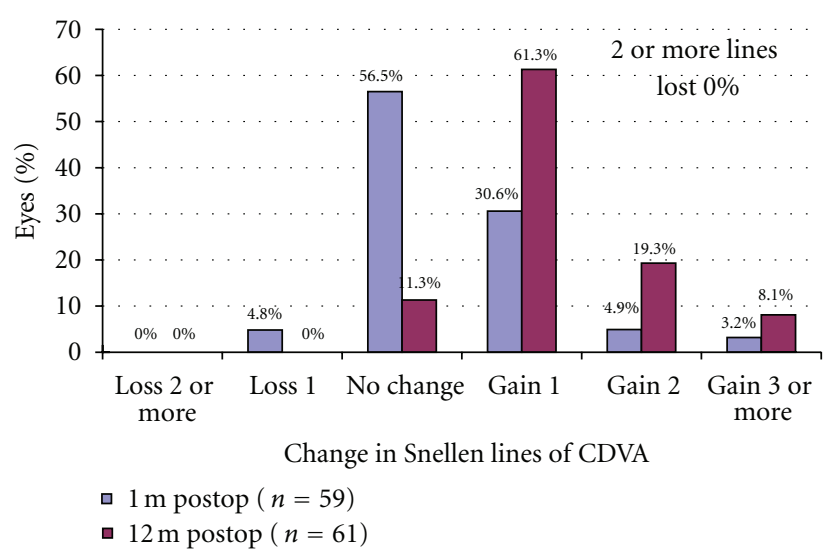

Figure 3: Gain/loss of CDVA 1 months and 12 months after epithelium-on CXL.

presentation purposes. Pre- and postoperative topography was analyzed using Precisio's irregularity index (IRI) as well as by measuring the central $5 \mathrm{~mm}$ using OPD indices. Statistical analysis was performed to compare the postoperative data with the preoperative data using the paired $t$-test with IBM SPSS Statistics v19.0 (IBM, Armonk, NY). $P<0.05$ was considered statistically significant.

\section{Results}

Sixty-one eyes of 53 patients fulfilled the inclusion and exclusion criteria. The mean age of the patients was $32 \pm 10$ years (range, $15-52$ years). $85 \%$ of the eyes (52 eyes) were from male patients.

3.1. Visual Acuity. Figures 2, 3, 4, and 5 and Table 1 show the visual acuity and refraction measurements pre- and postoperatively. The UDVA and CDVA improved significantly $(P<0.05)$. At 12 -month followup, none of the eyes lost lines of CDVA, while $27.4 \%$ of the eyes gained 2 or more lines and the safety index was 1.14 . At the same time point, mean spherical equivalent refraction decreased by $0.74 \mathrm{D}$ 


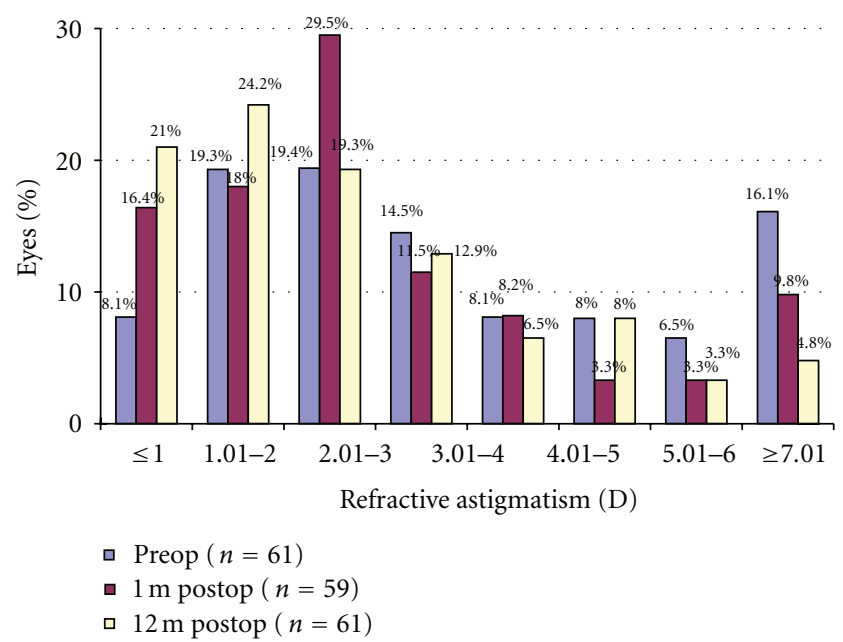

FIgURE 4: Refractive astigmatism after epithelium-on CXL.

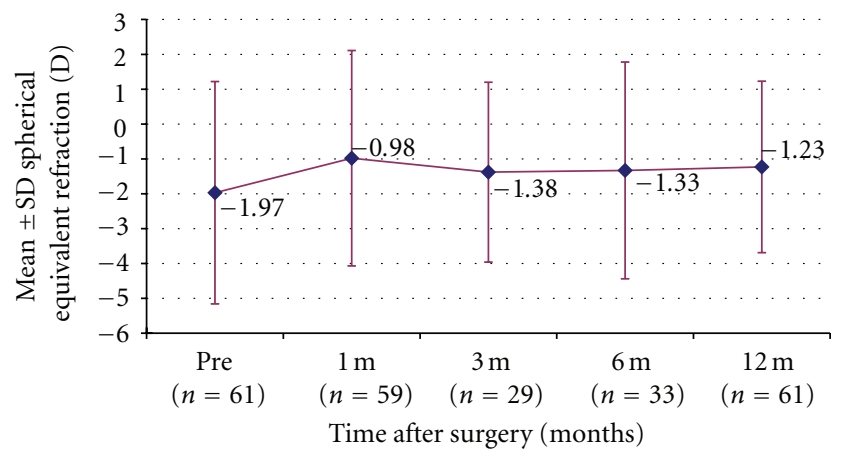

Figure 5: Stability of SE after epithelium-on CXL.

(less myopic, $P=0.05$ ), while mean cylinder decreased by $1.15 \mathrm{D}(P=0.00)$.

3.2. Corneal Topography and Wavefront Aberrometry. Table 2 shows the postoperative changes of topography and aberrometry. Data from the Precisio showed reduction in posterior elevation $(P=0.01)$, irregularity index $(P=0.01)$, and asymmetry $(P=0.01)$. The $K$-value from OPD did not significantly alter regarding Mean- $K$, while the Max- $K$ showed a significant decrease $(P=0.02)$ at the location of the cone. Figure 6 shows an example of the topographic changes in one of the treated eyes.

Aberrometry data showed a reduction in odd-order- $S$ $3,5,7(P=0.04)$ and total higher-order-aberrations $(P=$ $0.05)$.

3.3. Pachymetry. Precisio-measured pachymetry in Table 1 shows decrease in thickness at 1-month followup $(P=0.00)$ and thereafter a gradual increase to preoperative level at 12 months after the treatment $(P=0.15)$.
3.4. Pain Evaluation. Patients reported moderate to severe postoperative pain during the first 4-12 hours, peeking at 46 hours after surgery.

3.5. Complications. Discreet superficial epithelial layer damage could be observed on slit-lamp examination upon the verification of riboflavin saturation and after the CXL treatment. No serious complications were recorded during the follow-up period.

\section{Discussion}

Previous studies report conflicting results on the effects of epithelium-on CXL. While Pinelli and colleagues reported no significant difference in the analyzed parameters between epithelium-on and standard CXL [28], Wollensak and Iomdina found that the corneal biomechanical stiffening after epithelium-on CXL was about one-fifth compared to the epithelium-off CXL in an animal model [4]. Other clinical and laboratory studies have reported weaker or no effect of CXL using the epithelium-on method [22, 23, 25, 2931 . Collectively, the studies suggested that the significantly weaker biomechanical effect of epithelium-on CXL was due to the insufficient and inhomogeneous transepithelial riboflavin diffusion into the corneal stroma. However, a limitation of most of the studies that procured the low crosslinking effect or low stromal saturation of riboflavin with the epithelium-on CXL includes the use of the standard—or only slightly modified-Wollensak/Seiler protocol on nondeepithelialized eyes. Moreover, the authors did not attempt to enhance the riboflavin penetration, effectively only showing that the epithelium-on CXL does not work with the standard epithelium-off protocol. Intriguingly, epithelial permeability can be enhanced by application of several tensioactive substances including BAC and gentamicin at concentrations normally used in industrial preparations [32]. Such pharmacological enhancements, which are commonly used in epithelium-on CXL $[20,33]$, were included in the current protocol.

On the basis of previous studies reporting increased epithelial riboflavin permeability using hypotonic solution compared to isotonic solution $[24,27,34]$, hypotonic solution was applied in the current protocol. Furthermore, we avoided the use of riboflavin solution with dextran due to its high viscosity, which inhibits the penetration through the epithelium [35]. Hypotonic riboflavin was originally used with epithelium-off CXL protocol to induce significant edema in corneas thinner than $400 \mu \mathrm{m}$ [27]. However, the swelling of the corneas with intact epithelium seems to be of a considerably lower degree. In the present study, only around 10 microns of swelling were recorded in a subgroup of 29 eyes, presenting respective mean pachymetry of $450.50 \pm 42.90$ and $471.65 \pm 41.43 \mu \mathrm{m}$ before and after the corneal saturation with the $0.5 \%$ hypotonic riboflavin solution. Even though the clinical safety of CXL with hypotonic riboflavin solution has been documented [36], issues of the corneal endothelial cell toxicity were considered because of the decreased UV-protective effect with 
TABLE 1: Visual acuity, refraction, and corneal thickness changes during 1-year followup.

\begin{tabular}{|c|c|c|c|c|c|}
\hline \multirow{2}{*}{ Parameter } & \multirow{2}{*}{ Preoperative $(n=61)$} & \multicolumn{4}{|c|}{ Postoperative } \\
\hline & & 1 Month $(n=59)$ & 3 Months $(n=29)$ & 6 Months $(n=33)$ & 12 Months $(n=61)$ \\
\hline \multicolumn{6}{|c|}{ VA (Snellen) } \\
\hline UDVA & $(20 / 133 \pm 20 / 57)$ & $\begin{array}{c}(20 / 95 \pm 20 / 49) \\
(P=0.00)\end{array}$ & $\begin{array}{c}(20 / 87 \pm 20 / 44) \\
(P=0.00)\end{array}$ & $\begin{array}{c}(20 / 80 \pm 20 / 48) \\
(P=0.00)\end{array}$ & $\begin{array}{c}(20 / 67 \pm 20 / 42) \\
\quad(P=0.00)\end{array}$ \\
\hline CDVA & $(20 / 32 \pm 20 / 33)$ & $\begin{array}{c}(20 / 29 \pm 20 / 30) \\
\quad(P=0.01)\end{array}$ & $\begin{array}{c}(20 / 28 \pm 20 / 31) \\
\quad(P=0.00)\end{array}$ & $\begin{array}{c}(20 / 26 \pm 20 / 30) \\
\quad(P=0.00)\end{array}$ & $\begin{array}{c}(20 / 24 \pm 20 / 28) \\
\quad(P=0.00)\end{array}$ \\
\hline \multicolumn{6}{|c|}{ Refraction (D) } \\
\hline Sphere & $0.05 \pm 3.03$ & $\begin{array}{c}0.64 \pm 3.14 \\
(P=0.12)\end{array}$ & $\begin{array}{c}0.44 \pm 2.82 \\
(P=0.55)\end{array}$ & $\begin{array}{c}0.50 \pm 2.92 \\
(P=0.10)\end{array}$ & $\begin{array}{c}0.21 \pm 2.43 \\
(P=0.61)\end{array}$ \\
\hline SE & $-1.97 \pm 3.19$ & $\begin{array}{c}-0.98 \pm 3.09 \\
(P=0.00)\end{array}$ & $\begin{array}{c}-1.38 \pm 2.58 \\
(P=0.03)\end{array}$ & $\begin{array}{c}-1.33 \pm 3.11 \\
(P=0.01)\end{array}$ & $\begin{array}{c}-1.23 \pm 2.46 \\
(P=0.05)\end{array}$ \\
\hline Cylinder & $-4.03 \pm 2.53$ & $\begin{array}{c}-3.27 \pm 2.21 \\
(P=0.00)\end{array}$ & $\begin{array}{c}-3.65 \pm 2.62 \\
(P=0.01)\end{array}$ & $\begin{array}{c}-3.66 \pm 2.41 \\
(P=0.00)\end{array}$ & $\begin{array}{c}-2.88 \pm 2.00 \\
(P=0.00)\end{array}$ \\
\hline $\mathrm{CCT}(\mu \mathrm{m})$ & $451 \pm 45$ & $\begin{array}{c}425 \pm 58 \\
(P=0.00 ; n=21)\end{array}$ & $\begin{array}{c}436 \pm 45 \\
(P=0.00 ; n=31)\end{array}$ & $\begin{array}{c}441 \pm 58 \\
(P=0.81 ; n=26)\end{array}$ & $\begin{array}{c}460 \pm 47 \\
(P=0.15 ; n=50)\end{array}$ \\
\hline
\end{tabular}

UDVA: uncorrected distance visual acuity; CDVA: corrected distance visual acuity; SE: spherical equivalent; CCT: central corneal thickness.

TABLE 2: Topographic changes during 1-year followup.

\begin{tabular}{lcc}
\hline Parameter & Preoperative $(n=61)$ & 12 m postoperatively $(n=61)$ \\
\hline Precisio & & \\
PE $(\mu \mathrm{m})$ & $71.56 \pm 31.31$ & $66.48 \pm 28.81(P=0.01)$ \\
IRI $(\mu \mathrm{m})$ & $45.45 \pm 22.60$ & $42.18 \pm 22.54(P=0.01)$ \\
Asym $(\mathrm{D})$ & $9.05 \pm 5.50$ & $8.12 \pm 5.58(P=0.01)$ \\
OPD & & \\
Mean $K(\mathrm{D})$ & $46.97 \pm 5.21$ & $46.77 \pm 5.31(P=0.06)$ \\
Max K (D) & $55.55 \pm 6.01$ & $54.98 \pm 5.78(P=0.02)$ \\
DSI & $10.54 \pm 5.30$ & $9.71 \pm 5.01(P=0.00)$ \\
S 3, 5, 7 & $1.40 \pm 0.80$ & $1.32 \pm 0.80(P=0.04)$ \\
S 4, 6, 8 & $0.33 \pm 0.21$ & $0.34 \pm 0.33(P=0.81)$ \\
Total HOA & $4.80 \pm 2.93$ & $4.54 \pm 2.72(P=0.05)$ \\
\hline
\end{tabular}

PE: posterior elevation; IRI: irregularity index; Asym: asymmetry within $5 \mathrm{~mm}$ zone; DSI: differential sector index; HOA: higher order aberration.

hypotonic riboflavin due to the decrease of UV absorption coefficient from $\approx 53 \mathrm{~cm}^{-1}$ for $0.1 \%$ isotonic Riboflavin solution to $\approx 42 \mathrm{~cm}^{-1}$ for $0.1 \%$ hypotonic riboflavin solution [36]. To compensate for this, increasing the concentration of riboflavin in the hypoosmolar solution may enhance UVA absorption [37] and hence decrease the UV-radiation at the endothelial level. The current study addresses the endothelial safety as hypotonic riboflavin concentration of $0.5 \%$ was applied. A secondary benefit of the increased concentration is the presumably increased availability of the riboflavin molecules to penetrate the epithelium and saturate the stroma. In a subgroup analysis of 21 eyes performing pre- and postoperative specular microscopy using Konan CellCheck XL (Konan Medical, Irvine, CA), the endothelial count decreased insignificantly $(P=0.09)$ from $2738 \pm$ 188 cells $/ \mathrm{mm}^{2}$ to $2608 \pm 311$ cells $/ \mathrm{mm}^{2}$.

The current protocol also employed mechanical scarification of the epithelial surface by creation of microabrasions caused by movement of a Merocel sponge over the corneal surface with patient's blinking. The amount of such microabrasions could obviously not be standardized, and it varied between cases. This may be the reason for a relatively large variation in saturation time (mentioned in the next paragraph).

Finally, in addition to the chemical and mechanical enhancements, the current protocol demanded a slit-lamp verification of the stromal saturation before the UVA radiation (Figure 1). Our clinical observations showed that riboflavin saturation was achieved after 25-45 minutes, so that a commonly used set induction time of, for example, 30 minutes would in many cases lead to insufficient riboflavin concentration in the stroma.

Our visual and refractive outcomes were comparable to other published CXL studies. In the current study there were no cases with a loss of $\geq 2$ lines of CDVA, endothelial cell count did not change significantly, and there were no infections or other types of keratitis.

Most of the patients reported pain peaking 4-6 hours after the treatment despite the mostly preserved epithelium. This may be explained by actinic-keratoconjunctivitis-like reaction caused by the UV exposure during the treatment 


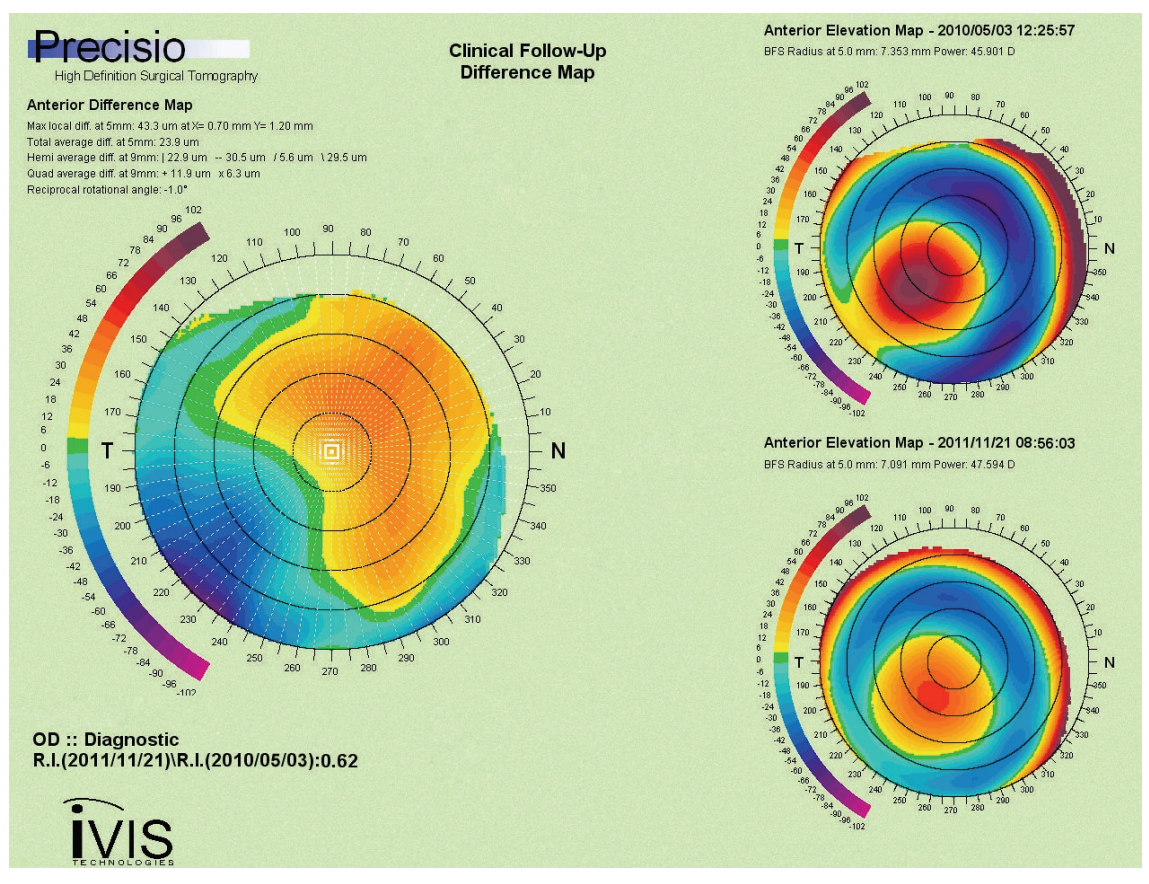

FIGURE 6: Scheimpflug anterior elevation difference map showing depression at the cone and increased elevation orthogonally (left image). Scheimpflug anterior elevation maps (right images): preoperative (upper) and 12-months after epithelium-on CXL (lower).

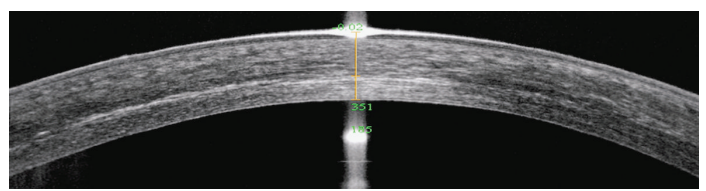

FIGURE 7: AS-OCT image showing demarcation line at $351 \mu \mathrm{m}, 2$ months after epithelium-on CXL.

and by the microabrasions caused on purpose, to enhance riboflavin penetration.

Corneal topography change in curvature has often been used to evaluate the effect of CXL $[23,38,39]$. In our study, the maximum- $K$ value and DSI (differential sector index) decreased significantly on the OPD II, Placido-based topography, as did the posterior elevation, irregularity index and asymmetry on the Precisio, Scheimpflug-based topography. However, our mean- $K$ did not decrease in contrast to most other studies. In most of our cases, in addition to the Max- $K$ decrease, a moderate increase in steepness on the opposite side of the cone occurred (Figure 6), resulting in only minor decrease of the mean- $K$ but more symmetrical corneal optics, decreased higher-order-aberrations, and improved vision. We hypothesize that this may be a consequence of a possibly heavier riboflavin load in the inferior corneal stroma due to the sitting position and blinking during the riboflavin induction, leading to a locally increased cross-linking effect. This theory warrants a further study and may be a small step in the direction of "customized" CXL.
Detection of the demarcation line [40] after CXL has been considered the proof of the efficacy and the measure of the depth of the corneal cross-linking. Although the precise nature and significance of the demarcation line (increased optical density) in relation to the cross-linking process are uncertain, it may be consequent to the keratocyte apoptosis and their subsequent repopulation [41]. Keratocyte apoptosis has to a lesser extent been demonstrated after epitheliumon CXL [4]. Filippello et al. epithelium-on CXL with 0.1\% isotonic riboflavin solution showed that the demarcation line two weeks postoperatively was located approximately $100 \mu \mathrm{m}$ from the corneal epithelium [23]. In 24 eyes treated with the current protocol that could be evaluated by RTVue (Optovue Inc., Fremont, CA) AS-OCT (anterior segment optical coherence tomography), the mean demarcation line was located at the depth of $316.92 \pm 49.16 \mu \mathrm{m}$ (range 260 to 367 ) from the surface (Figure 7), which is close to the observations after epithelium-off CXL.

Epithelial absorption/filtering of the UVA light that could potentially lead to lesser energy delivered to riboflavinsaturated stroma has also been stated as an argument against the use of epithelium-on CXL. Different studies offer a variety of evaluation backgrounds of this matter. A study performed by Baiocci et al. [21] claimed that human corneal epithelium and the underlying basement membrane naturally absorb $30 \%$ to $33 \%$ of UVA radiation (400 to $350 \mathrm{~nm}$ ), while other studies showed that the epithelial UV absorption occurs only with wavelengths lower than $310 \mathrm{~nm}$ [42-44]. We may assume that the UV-absorption of the riboflavin within the epithelium is probably low (since the epithelial cells are hydrophobic and do not absorb 
riboflavin) and that the epithelial interstitial space is of negligible volume. The current protocol includes washing off the riboflavin from the corneal surface before the UVAradiation in order to minimize the UV-energy loss due to its possible absorption by the riboflavin.

Finally, even if the epithelium-on CXL leads to a shallower cross-linking compared to the epithelium-off, the density of collagen fibers in corneal stroma is much higher in the anterior portion where most of the collagen cross-links occur $[40,45]$.

\section{Conclusion}

This retrospective study showed that epithelium-on CXL using our novel protocol appeared to be effective and safe in treating progressive keratoconus. The improvements in the visual, refractive, and topographic parameters in our patients indicate that epithelium-on CXL had sufficient effect to halt the progression of keratoconus and improve the corneal shape. A randomized controlled trial is warranted to verify that the effect of the current approach is comparable with the standard epithelium-off CXL. Furthermore, the combination of different enhancers, osmolality, and concentration of riboflavin should be explored to further improve the procedure.

\section{Conflict of Interests}

None of the authors has a commercial, proprietary, or financial interest in any material or procedure mentioned.

\section{References}

[1] L. Spadea, "Collagen crosslinking for ectasia following PRK performed in excimer laser-assisted keratoplasty for keratoconus," European Journal of Ophthalmology, vol. 22, no. 2, pp. 274-277, 2012.

[2] C. Mazzotta, A. Balestrazzi, C. Traversi et al., "Treatment of progressive keratoconus by riboflavin-UVA-induced crosslinking of corneal collagen: ultrastructural analysis by Heidelberg Retinal Tomograph II in vivo confocal microscopy in humans," Cornea, vol. 26, no. 4, pp. 390-397, 2007.

[3] F. Hafezi, J. Kanellopoulos, R. Wiltfang, and T. Seiler, "Corneal collagen crosslinking with riboflavin and ultraviolet A to treat induced keratectasia after laser in situ keratomileusis," Journal of Cataract \& Refractive Surgery, vol. 33, no. 12, pp. 2035-2040, 2007.

[4] G. Wollensak and E. Iomdina, "Biomechanical and histological changes after corneal crosslinking with and without epithelial debridement," Journal of Cataract \& Refractive Surgery, vol. 35, no. 3, pp. 540-546, 2009.

[5] G. Wollensak, M. Wilsch, E. Spoerl, and T. Seiler, "Collagen fiber diameter in the rabbit cornea after collagen crosslinking by riboflavin/UVA," Cornea, vol. 23, no. 5, pp. 503-507, 2004.

[6] E. Spoerl, G. Wollensak, and T. Seiler, "Increased resistance of crosslinked cornea against enzymatic digestion," Current Eye Research, vol. 29, no. 1, pp. 35-40, 2004.

[7] C. Mazzotta, C. Traversi, S. Baiocchi et al., "Corneal healing after riboflavin ultraviolet-A collagen cross-linking determined by confocal laser scanning microscopy in vivo: early and late modifications," American Journal of Ophthalmology, vol. 146, no. 4, pp. 527-533, 2008.

[8] G. Wollensak and B. Redl, "Gel electrophoretic analysis of corneal collagen after photodynamic cross-linking treatment," Cornea, vol. 27, no. 3, pp. 353-356, 2008.

[9] C. Mazzotta, T. Caporossi, R. Denaro et al., "Morphological and functional correlations in riboflavin UV A corneal collagen cross-linking for keratoconus," Acta Ophthalmologica, vol. 90, no. 3, pp. 259-265, 2012.

[10] G. Wollensak, E. Spoerl, M. Wilsch, and T. Seiler, "Endothelial cell damage after riboflavin-ultraviolet-A treatment in the rabbit," Journal of Cataract \& Refractive Surgery, vol. 29, no. 9, pp. 1786-1790, 2003.

[11] E. Spoerl, M. Mrochen, D. Sliney, S. Trokel, and T. Seiler, "Safety of UVA-riboflavin cross-linking of the cornea," Cornea, vol. 26, no. 4, pp. 385-389, 2007.

[12] M. Ahearne, Y. Yang, K. Y. Then, and K. K. Liu, "Nondestructive mechanical characterisation of UVA/riboflavin crosslinked collagen hydrogels," The British Journal of Ophthalmology, vol. 92, no. 2, pp. 268-271, 2008.

[13] G. Wollensak, E. Spoerl, and T. Seiler, "Riboflavin/ultravioletA-induced collagen crosslinking for the treatment of keratoconus," American Journal of Ophthalmology, vol. 135, no. 5, pp. 620-627, 2003.

[14] A. Caporossi, S. Baiocchi, C. Mazzotta, C. Traversi, and T. Caporossi, "Parasurgical therapy for keratoconus by riboflavin-ultraviolet type A rays induced cross-linking of corneal collagen: preliminary refractive results in an Italian study," Journal of Cataract \& Refractive Surgery, vol. 32, no. 5, pp. 837-845, 2006.

[15] D. S. Grewal, G. S. Brar, R. Jain, V. Sood, M. Singla, and S. P. S. Grewal, "Corneal collagen crosslinking using riboflavin and ultraviolet-A light for keratoconus: one-year analysis using Scheimpflug imaging," Journal of Cataract \& Refractive Surgery, vol. 35, no. 3, pp. 425-432, 2009.

[16] K. V. Zamora and J. J. Males, "Polymicrobial keratitis after a collagen cross-linking procedure with postoperative use of a contact lens: a case report," Cornea, vol. 28, no. 4, pp. 474-476, 2009.

[17] J. J. Pérez-Santonja, A. Artola, J. Javaloy, J. L. Alió, and J. L. Abad, "Microbial keratitis after corneal collagen crosslinking," Journal of Cataract \& Refractive Surgery, vol. 35, no. 6, pp. 1138-1140, 2009.

[18] C. Mazzotta, A. Balestrazzi, S. Baiocchi, C. Traversi, and A. Caporossi, "Stromal haze after combined riboflavin-UVA corneal collagen cross-linking in keratoconus: in vivo confocal microscopic evaluation," Clinical \& Experimental Ophthalmology, vol. 35, no. 6, pp. 580-582, 2007.

[19] P. Eberwein, C. Auw-Hädrich, F. Birnbaum, P. C. Maier, and T. Reinhard, "Corneal melting after cross-linking and deep lamellar keratoplasty in a keratokonus patient," Klinische Monatsblatter für Augenheilkunde, vol. 225, no. 1, pp. 96-98, 2008.

[20] B. S. Boxer Wachler, R. Pinelli, A. Ertan, and C. C. K. Chan, "Safety and efficacy of transepithelial crosslinking (C3R/CXL)," Journal of Cataract \& Refractive Surgery, vol. 36, no. 1, pp. 186-189, 2010.

[21] S. Baiocchi, C. Mazzotta, D. Cerretani, T. Caporossi, and A. Caporossi, "Corneal crosslinking: riboflavin concentration in corneal stroma exposed with and without epithelium," Journal of Cataract \& Refractive Surgery, vol. 35, no. 5, pp. 893-899, 2009.

[22] K. Samaras, D. P. O’Brart, J. Doutch, S. Hayes, J. Marshall, and K. M. Meek, "Effect of epithelial retention and removal on 
riboflavin absorption in porcine corneas," Journal of Refractive Surgery, vol. 25, no. 9, pp. 771-775, 2009.

[23] M. Filippello, E. Stagni, and D. O’Brart, "Transepithelial corneal collagen crosslinking: bilateral study," Journal of Cataract \& Refractive Surgery, vol. 38, no. 2, pp. 283-291, 2012.

[24] A. Kissner, E. Spoerl, R. Jung, K. Spekl, L. E. Pillunat, and F. Raiskup, "Pharmacological modification of the epithelial permeability by benzalkonium chloride in UVA/Riboflavin corneal collagen cross-linking," Current Eye Research, vol. 35, no. 8, pp. 715-721, 2010.

[25] S. Hayes, D. P. O’Brart, L. S. Lamdin et al., "Effect of complete epithelial debridement before riboflavin-ultraviolet-A corneal collagen crosslinking therapy," Journal of Cataract \& Refractive Surgery, vol. 34, no. 4, pp. 657-661, 2008.

[26] E. F. Bakke, A. Stojanovic, X. Chen, and L. Drolsum, "Penetration of riboflavin and postoperative pain in corneal collagen crosslinking: excimer laser superficial versus mechanical fullthickness epithelial removal," Journal of Cataract \& Refractive Surgery, vol. 35, no. 8, pp. 1363-1366, 2009.

[27] F. Raiskup and E. Spoerl, "Corneal cross-linking with hypoosmolar riboflavin solution in thin keratoconic corneas," American Journal of Ophthalmology, vol. 152, no. 1, pp. 28.e132.e1, 2011.

[28] R. Pinelli, M. M. Al Marzouky, and H. I. El-Shawaf, "Tensioactive-mediated transepithelial corneal crosslinking-first laboratory report," European Ophthalmic Review, vol. 3, no. 2, pp. 67-70, 2009.

[29] K. M. Bottós, J. L. Dreyfuss, C. V. S. Regatieri et al., "Immunofluorescence confocal microscopy of porcine corneas following collagen cross-linking treatment with riboflavin and ultraviolet A," Journal of Refractive Surgery, vol. 24, no. 7, pp. S715-S719, 2008.

[30] K. E. Samaras and D. B. Lake, "Corneal collagen cross linking (CXL): a review," International Ophthalmology Clinics, vol. 50, no. 3, pp. 89-100, 2010.

[31] A. Leccisotti and T. Islam, "Transepithelial corneal collagen cross-linking in keratoconus," Journal of Refractive Surgery, vol. 26, no. 12, pp. 942-948, 2010.

[32] S. H. Cha, J. S. Lee, B. S. Oum, and C. D. Kim, "Corneal epithelial cellular dysfunction from benzalkonium chloride (BAC) in vitro," Clinical \& Experimental Ophthalmology, vol. 32, no. 2, pp. 180-184, 2004.

[33] B. Boxer Wachler, "Corneal Collagen Crosslinking with Riboflavin. A new treatment to strengthen the cornea for keratoectasia and keratoconus," Cataract \& Refractive Surgery Today, vol. 1, pp. 73-74, 2005.

[34] F. Raiskup, R. Pinelli, and E. Spoerl, "Riboflavin osmolar modification for transepithelial corneal cross-linking," Current Eye Research, vol. 37, no. 3, pp. 234-238, 2012.

[35] D. L. Meadows, J. R. Paugh, A. Joshi, and J. Mordaunt, "A novel method to evaluate residence time in humans using a nonpenetrating fluorescent tracer," Investigative Ophthalmology \& Visual Science, vol. 43, no. 4, pp. 1032-1039, 2002.

[36] F. Hafezi, M. Mrochen, H. P. Iseli, and T. Seiler, "Collagen crosslinking with ultraviolet-A and hypoosmolar riboflavin solution in thin corneas," Journal of Cataract \& Refractive Surgery, vol. 35, no. 4, pp. 621-624, 2009.

[37] G. Wollensak, H. Aurich, C. Wirbelauer, and S. Sel, "Significance of the riboflavin film in corneal collagen crosslinking," Journal of Cataract \& Refractive Surgery, vol. 36, no. 1, pp. 114 120, 2010.

[38] D. Asri, D. Touboul, P. Fournie et al., "Corneal collagen crosslinking in progressive keratoconus: multicenter results from the French National Reference Center for Keratoconus,"
Journal of Cataract \& Refractive Surgery, vol. 37, pp. 2137$2143,2011$.

[39] P. Vinciguerra, E. Albè, S. Trazza et al., "Refractive, topographic, tomographic, and aberrometric analysis of keratoconic eyes undergoing corneal cross-linking," Ophthalmology, vol. 116, no. 3, pp. 369-378, 2009.

[40] T. Seiler and F. Hafezi, "Corneal cross-linking-induced stromal demarcation line," Cornea, vol. 25, no. 9, pp. 1057-1059, 2006.

[41] G. Wollensak, E. Iomdina, D. D. Dittert, and H. Herbst, "Wound healing in the rabbit cornea after corneal collagen cross-linking with riboflavin and UVA," Cornea, vol. 26, no. 5, pp. 600-605, 2007.

[42] L. Kolozsvári, A. Nógrádi, B. Hopp, and Z. Bor, "UV absorbance of the human cornea in the 240- to 400-nm range," Investigative Ophthalmology \& Visual Science, vol. 43, no. 7, pp. 2165-2168, 2002.

[43] A. Ringvold, "Corneal epithelium and UV-protection of the eye," Acta Ophthalmologica Scandinavica, vol. 76, no. 2, pp. 149-153, 1998.

[44] A. Podskochy, "Protective role of corneal epithelium against ultraviolet radiation damage," Acta Ophthalmologica Scandinavica, vol. 82, no. 6, pp. 714-717, 2004.

[45] G. Wollensak, E. Spoerl, and T. Seiler, "Stress-strain measurements of human and porcine corneas after riboflavinultraviolet-A-induced cross-linking," Journal of Cataract \& Refractive Surgery, vol. 29, no. 9, pp. 1780-1785, 2003. 


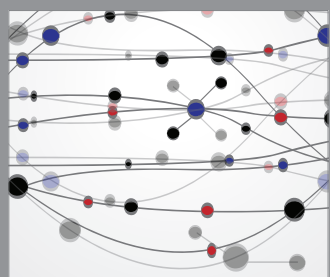

The Scientific World Journal
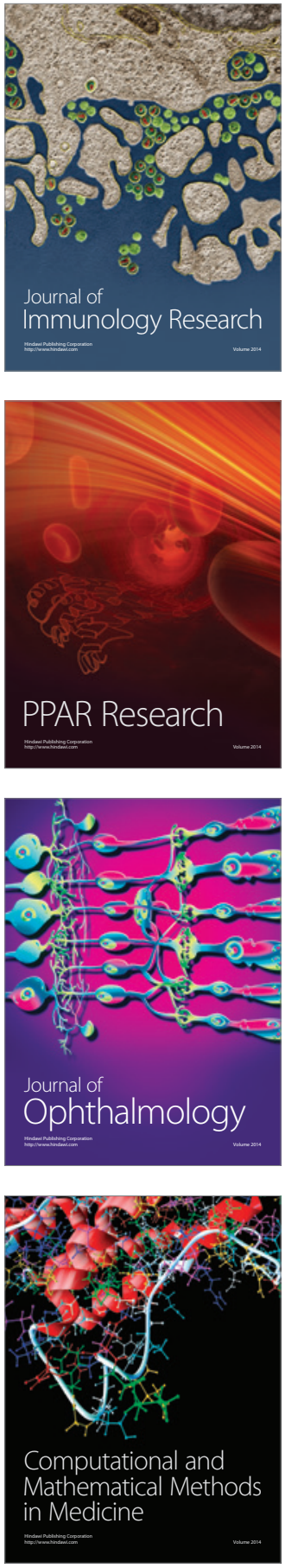

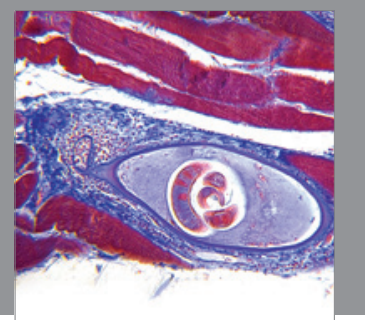

Gastroenterology

Research and Practice
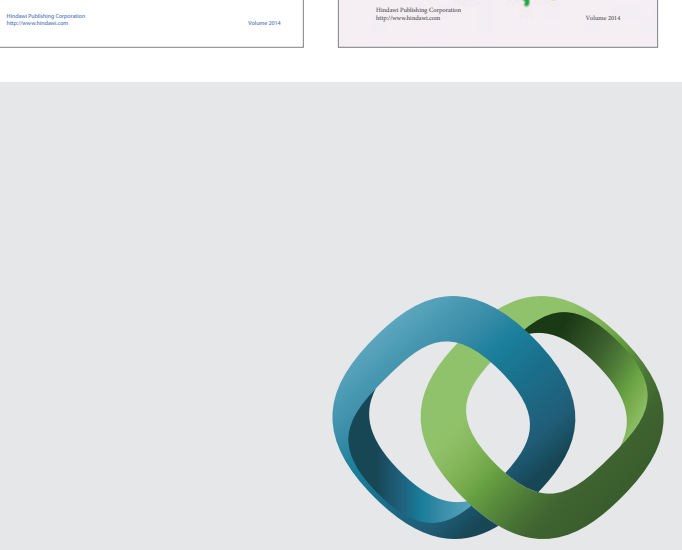

\section{Hindawi}

Submit your manuscripts at

http://www.hindawi.com
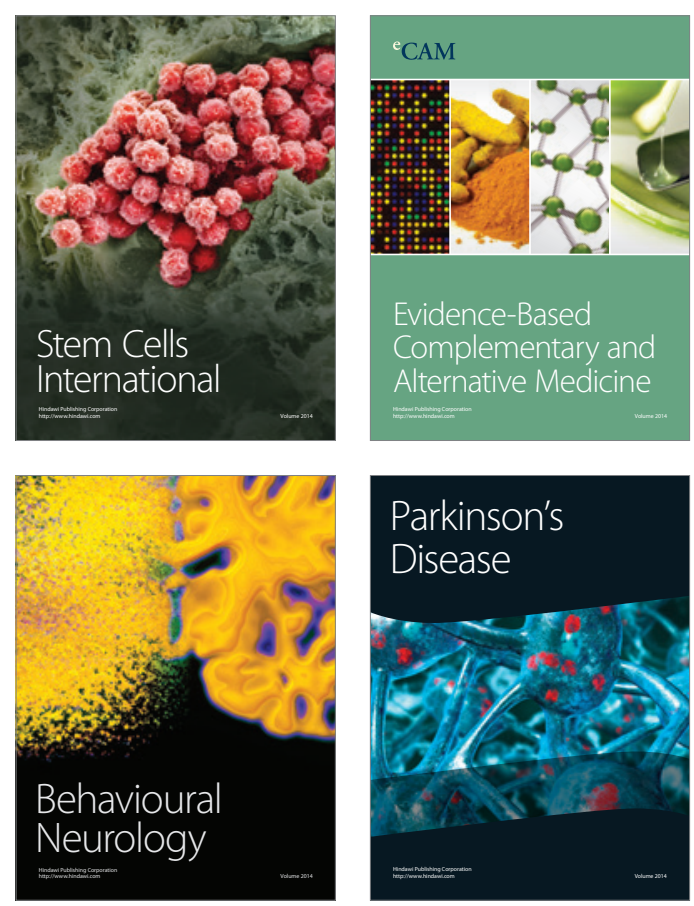

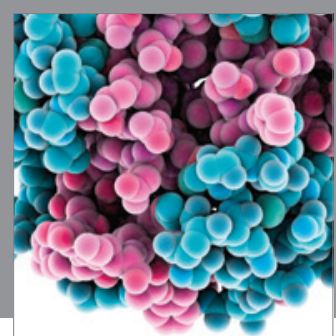

Journal of
Diabetes Research

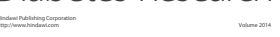

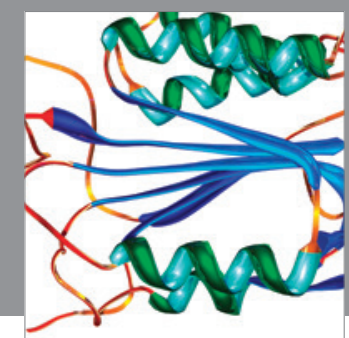

Disease Markers
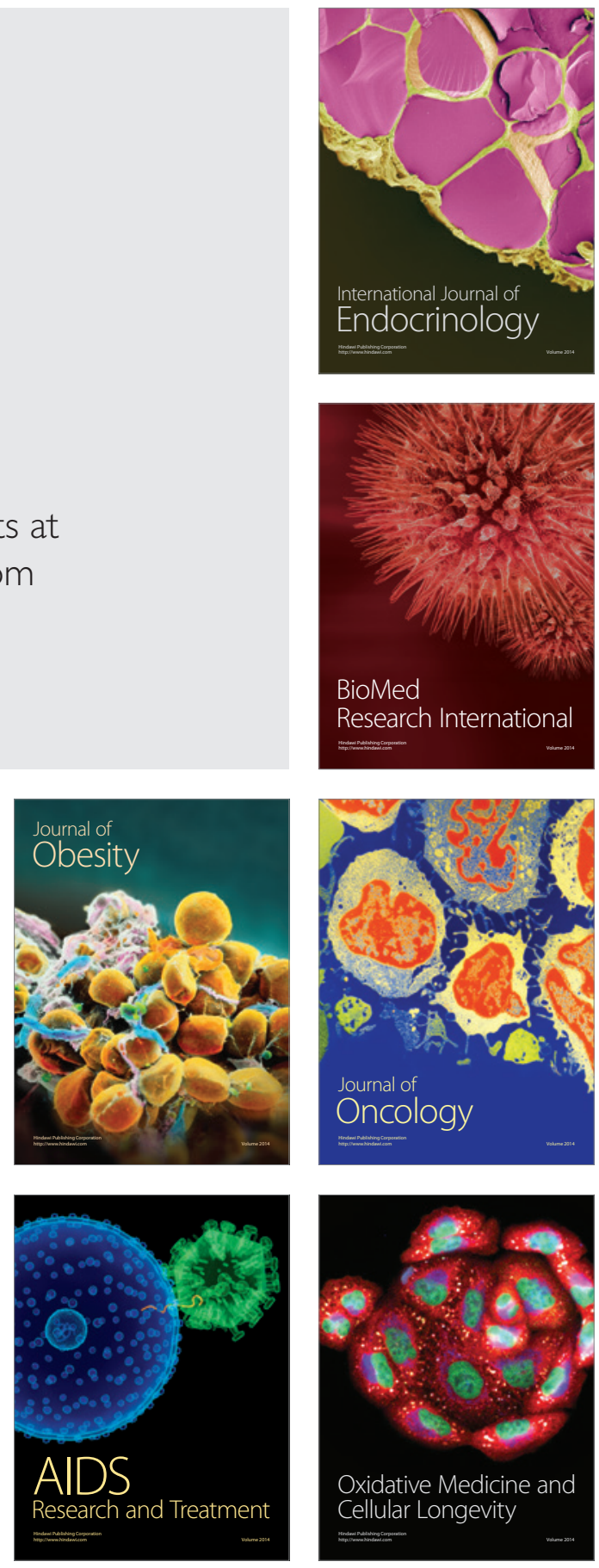